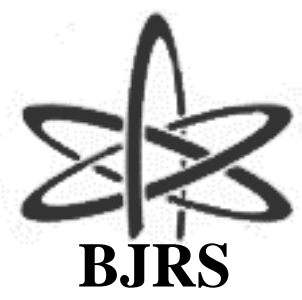

\author{
BRAZILIAN JOURNAL \\ $\mathrm{OF}$ \\ RADIATION SCIENCES \\ 08-02 (2020) 01-18
}

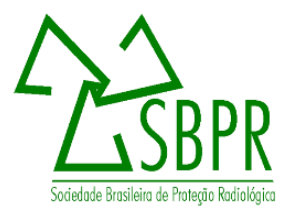

\title{
Investigação da termoluminescência de alexandrita $\left(\mathrm{BeAl}_{2} \mathrm{O}_{4}: \mathrm{Cr}^{3+}\right)$
}

\author{
Dardengo $^{a}$ S. L., Nunes ${ }^{a}$ M. C. S., Ulsen ${ }^{b}$ C., Yoshimura $^{c}$ E. M., Trindade ${ }^{\mathrm{a}, \mathrm{c}}$ N. M. \\ ${ }^{a}$ Departamento de Física, Instituto Federal de Educação, Ciência e Tecnologia de São Paulo, Rua Pedro Vicente, 625, \\ Canindé, São Paulo, SP, Brasil.
}

${ }^{b}$ Escola Politécnica da Universidade de São Paulo, Laboratório de Caracterização Tecnológica, Av. Professor Mello Moraes, 2373, Cidade Universitária, São Paulo, SP, Brasil.

${ }^{c}$ Instituto de Física, Universidade de São Paulo, Rua do Matão, 1371, Cidade Universitária, São Paulo, SP, Brasil.

ntrindade@ifsp.edu.br

\section{RESUMO}

O mineral alexandrita $\left(\mathrm{BeAl}_{2} \mathrm{O}_{4}: \mathrm{Cr}^{3+}\right)$ é amplamente encontrado no Brasil e tem sido investigado para o emprego como um detector de radiação ionizante devido as suas propriedades termoluminescentes (TL). A utilização de materiais naturais na área é interessante pelo seu menor custo comparado aos sintéticos, além da possibilidade de atuarem na dosimetria retrospectiva. Diferentes características das curvas TL de sete amostras foram investigadas, como a linearidade, repetibilidade e fading. As amostras foram irradiadas com doses entre 0,5 Gy e 5 Gy, utilizando uma fonte beta de ${ }^{90} \mathrm{Sr} /{ }^{90} \mathrm{Y}$. A deconvolução da curva de emissão TL por um modelo de cinética de primeira ordem revelou cinco picos nas temperaturas de $355 \mathrm{~K}, 405 \mathrm{~K}, 445 \mathrm{~K}, 530 \mathrm{~K}$ e $585 \mathrm{~K}$ (taxa de aquecimento de $1 \mathrm{~K} / \mathrm{s}$ ). Os resultados demonstram que a resposta TL é linear com a dose, tem boa repetibilidade e o sinal é estável à temperatura ambiente por até 33 dias de armazenamento após a irradiação. A caracterização química constatou que as amostras utilizadas são predominantemente formadas pela fase alexandrita com uma porção significativa de fases de apatita. Em geral, os resultados das características estudadas mostraram que a alexandrita tem potencial de aplicação na área de dosimetria.

Palavras-chave: alexandrita, mineral, termoluminescência, dosimetria. 


\begin{abstract}
Alexandrite mineral $\left(\mathrm{BeAl}_{2} \mathrm{O}_{4}: \mathrm{Cr}^{3+}\right)$, that is largely found in Brazil, is being investigated to be used as an ionizing radiation detector due to its thermoluminescent (TL) property. Natural materials to be used as dosimeters have a lower cost than synthetic ones and can be used also in retrospective dosimetry. Different TL characteristics of seven samples were studied, such as linearity, repeatability, and fading. The alexandrite samples were irradiated to doses of $0.5 \mathrm{~Gy}$ to $5 \mathrm{~Gy}$ using a ${ }^{90} \mathrm{Sr} /{ }^{90} \mathrm{Y}$ beta source. The deconvolution of the TL glow curve with a first order kinetic model showed five peaks at $355 \mathrm{~K}, 405 \mathrm{~K}, 445 \mathrm{~K}, 530 \mathrm{~K}$ and $585 \mathrm{~K}$ (heating rate $1 \mathrm{~K} / \mathrm{s}$ ). The results showed that the TL signal has a linear dose-response with good repeatability and that the signal is stable at room temperature at least till 33 days of storage after irradiation. The chemical microanalysis indicated that samples are predominantly composed of alexandrite grains and a significant content of apatite. In general, the TL results showed some of the desirable characteristics of dosimetric materials, suggesting that natural alexandrite have a potential application in this field.
\end{abstract}

Keywords: alexandrite, mineral, thermoluminescence, dosimetry.

\title{
1. INTRODUÇÃO
}

O Brasil é considerado o maior fornecedor de alexandrita $\left(\mathrm{BeAl}_{2} \mathrm{O}_{4}: \mathrm{Cr}^{3+}\right)$ no mundo, com as maiores concentrações de jazidas nos estados de Minas Gerais, Bahia e Espírito Santo [1]. Essa gema é uma variedade do mineral crisoberilo $\left(\mathrm{BeAl}_{2} \mathrm{O}_{4}\right)$. Uma de suas características é a capacidade de mudança de cor, sendo geralmente verde à luz natural, e vermelho à luz incandescente, fenômeno conhecido na literatura por "efeito alexandrita" [2]. Além disso, no que se refere às aplicações tecnológicas, uma das mais importantes aplicações da alexandrita se dá como meio ativo para laser de estado sólido, sendo primeiramente reportado por Bukin [3], possuindo diversas vantagens com relação a outros lasers, como os de Rubi $\left(\mathrm{Al}_{2} \mathrm{O}_{3}: \mathrm{Cr}^{3+}\right)$ e o Nd-YAG $\left(\mathrm{Y}_{3} \mathrm{Al}_{5} \mathrm{O}_{12}: \mathrm{Nd}^{3+}\right)$ [4]. Trabalhos abrangentes sobre as aplicações do laser alexandrita na medicina podem ser encontrados em [5-11]. Apesar dessas aplicações, há uma escassa literatura quando se refere às propriedades luminescentes de alexandrita voltadas ao campo da dosimetria.

Atualmente, há vários grupos de pesquisa no Brasil e no mundo preocupados com o desenvolvimento de materiais para dosimetria, assim como protocolos de utilização desses dosímetros [12]. Tais dosímetros são compostos por materiais detectores sensíveis à radiação, os 
quais, após serem submetidos a um estímulo térmico ou óptico, emitem luz com intensidade proporcional à dose de radiação absorvida pelo material [13]. No caso da termoluminescência, ocorre uma emissão termicamente estimulada originada da energia que foi previamente armazenada no cristal durante a irradiação [14,15]. A técnica de termoluminescência, por ser mais antiga, apresenta uma longa trajetória de sucesso, com diversas aplicações em dosimetria das radiações, especialmente na dosimetria pessoal [16]. Há minerais que apresentam características desejadas para dosimetria termoluminescente (TL), como dosimetria de acidentes nucleares, controle de irradiação de alimentos, e datação por luminescência [15,17]. Entre eles, o quartzo [18-20], a calcita $[21,22]$ e o feldspato [17,23] são minerais bem conhecidos; e, recentemente, concluiu-se que o mineral alexandrita $\left(\mathrm{BeAl}_{2} \mathrm{O}_{4}: \mathrm{Cr}^{3+}\right)$ apresenta potencial para uso em dosimetria [24,25].

O potencial da alexandrita como detector de radiação ionizante se deve principalmente a sua composição química, com cerca de $20 \%$ de $\mathrm{BeO}$ e $80 \%$ de $\mathrm{Al}_{2} \mathrm{O}_{3}$ [26], ambos sendo óxidos já comercializados como dosímetros passivos de estado sólido. $\mathrm{O} \mathrm{Al}_{2} \mathrm{O}_{3}$ é um dosímetro amplamente utilizado pela sua ótima sensibilidade, além de uma resposta linear para doses de até cerca de 10 Gy [13]. O óxido de berílio $(\mathrm{BeO})$ também é um ótimo material dosimétrico, demonstrando uma boa sensibilidade à radiação ionizante e possuindo uma resposta linear para uma grande faixa de variações de dose, além de ter um número atômico efetivo $\left(Z_{\mathrm{ef}}=7,2\right)$ próximo ao do tecido humano $\left(\mathrm{Z}_{\mathrm{ef}} \approx 7,6\right)$, sendo assim, utilizado principalmente na dosimetria médica e pessoal [13,27,28]. Outro fator importante, é que a alexandrita pode ser uma alternativa de menor custo em comparação com os materiais crescidos sinteticamente, além de ser amplamente encontrada em território brasileiro.

\section{MATERIAIS E MÉTODOS}

As amostras de alexandrita utilizadas nesse trabalho são provenientes do estado da Bahia (Brasil). Preliminarmente, foi realizado um tratamento térmico com um aquecimento de $20 \mathrm{~K} / \mathrm{min}$, até uma temperatura máxima de $673 \mathrm{~K}$ em um forno mufla (marca EDGCON, modelo 3000) sendo mantida a alexandrita nessa temperatura durante 1 hora para que o efeito da radiação ambiental (resultando no armazenamento de energia) fosse removido. Por se tratar de uma amostra natural e a fim de obter resultados mais homogêneos nas medidas de termoluminescência, optou-se por 
cominuir os fragmentos de alexandrita usando um almofariz e pistilo de ágata da marca Chiarotti até que toda amostra tivesse granulação menor que $0,35 \mathrm{~mm}$, controlada por peneiramento a seco. Os instrumentos utilizados foram devidamente higienizados com álcool isopropílico para que não houvesse contaminação entre as amostras. Para a realização das medidas de termoluminescência, o pó foi separado em 7 amostras, de forma que todos tivessem uma massa semelhante em cada experimento. As amostras foram denominadas $Q_{i}(i=1,2,3, \ldots, 7)$.

Nas medidas de termoluminescência foi utilizado o leitor comercial Ris $\varnothing$ (modelo DA-20). O sistema possui uma fonte radioativa beta de ${ }^{90} \mathrm{Sr} /{ }^{90} \mathrm{Y}(10 \mathrm{mGy} / \mathrm{s})$. Todos os experimentos foram realizados com uma máscara óptica de $5 \mathrm{~mm}$ de diâmetro e filtro UV Hoya U-340 (7,5 mm de espessura).

As medidas de termoluminescência foram realizadas a uma taxa de aquecimento de $1 \mathrm{~K} / \mathrm{s}$. Os experimentos foram divididos da seguinte maneira:

$\checkmark \quad T_{M}-T_{S T O P}$ : Os principais picos foram caracterizados utilizando o método $T_{M}-T_{S T O P}$ proposto por McKeever [29], que consiste em verificar a quantidade, bem como a posição de picos individuais presentes em curvas TL complexas. Para realizar essa caracterização, a amostra $Q_{6}$ previamente irradiada com $5 \mathrm{~Gy}$ foi aquecida $(\beta=1 \mathrm{~K} / \mathrm{s})$ até uma temperatura $T_{S T O P}$, sendo rapidamente resfriada até a temperatura ambiente. Então, a amostra foi reaquecida para verificar a curva TL restante. A partir dessa curva, a posição de temperatura do primeiro pico $T_{M}$ é verificada. Esse processo foi repetido diversas vezes, para valores crescentes de $T_{\text {STOP }}$ em intervalo de $10 \mathrm{~K}$, entre as temperaturas 443 a $673 \mathrm{~K}$. Para a análise, foi feito um gráfico de $T_{M}$ por $T_{S T O P}$, de forma que as posições de temperatura dos principais picos pudessem ser observadas.

$\checkmark$ Dose-resposta: As amostras $Q_{2}$ a $Q_{5}$ foram posicionadas arbitrariamente nos cups do leitor Ris $\emptyset$. Foram primeiramente tomadas as medidas do background (aquecimento sem irradiação prévia). Então, as amostras foram irradiadas com dose beta, variando de 0,5 a 5 Gy. As leituras TL foram realizadas na faixa de temperaturas entre 300 e $800 \mathrm{~K}$. O objetivo principal desse experimento foi verificar se há relação de linearidade entre as doses fornecidas e o sinal TL resultante.

$\checkmark$ Repetibilidade: Para esse experimento, a amostra $Q_{7}$ foi utilizada. Foram realizadas 5 me- 
didas com a mesma dose beta de 1 Gy. Por se tratar de uma amostra natural, o objetivo principal desse experimento foi verificar qual seria o grau de confiabilidade dos resultados das medidas de termoluminescência.

$\checkmark$ Fading: Nesse experimento, a amostra $Q_{1}$ foi irradiada com dose beta de 5 Gy, e após 2 e 33 dias (independentemente) a leitura TL foi realizada. Esse resultado foi comparado com a integral da curva TL obtida imediatamente após a exposição à radiação ionizante, a fim de compreender a estabilidade do sinal TL (picos mais intensos) da alexandrita natural.

Na Tabela 1 está um resumo, por amostra, em quais procedimentos essas foram utilizadas.

Tabela 1: Amostras $Q_{i}$ relacionadas aos procedimentos listados.

\begin{tabular}{ccc}
\hline Amostra & Procedimento & Massa $\left(\mathbf{1 0}^{-\mathbf{2}} \mathbf{g}\right)$ \\
\hline $\mathrm{Q}_{1}$ & Fading & $(2,7 \pm 0,1) \cdot$ \\
$\mathrm{Q}_{2}$ & Dose-resposta & $(2,5 \pm 0,1)$ \\
$\mathrm{Q}_{3}$ & Dose-resposta & $(2,5 \pm 0,1)$ \\
$\mathrm{Q}_{4}$ & Dose-resposta & $(2,5 \pm 0,1)$ \\
$\mathrm{Q}_{5}$ & Dose-resposta & $(2,5 \pm 0,1)$ \\
$\mathrm{Q}_{6}$ & $\mathrm{~T}_{\mathrm{M}-\mathrm{T}}-\mathrm{T}_{\mathrm{STOP}}$ & $(2,4 \pm 0,1)$ \\
$\mathrm{Q}_{7}$ & Repetibilidade & $(3,3 \pm 0,1)$ \\
\hline
\end{tabular}

Para a análise das curvas TL, foi utilizado o software GlowFit [30], desenvolvido para a plataforma MS Windows, programado na linguagem $\mathrm{C}++$. O software faz a deconvolução das curvas a partir dos dados experimentais, considerando os parâmetros de taxa de aquecimento e número de picos presentes. A deconvolução é baseada no modelo matemático de Randall \& Wilkins (1945) [31], que descreve curvas TL que seguem o mecanismo cinético de primeira ordem. O software ainda fornece informações como posições, intensidades, áreas e energias dos picos TL [30]. A qualidade desses ajustes é verificada pelo parâmetro figure of merit (FOM). De acordo com a literatura [32-35], o parâmetro FOM é considerado bom quando menor do que 5\%.

Algumas partículas da amostra analisada foram caracterizadas por microscopia eletrônica de varredura (MEV), com sistema de microanálise química por dispersão de energia (EDS); as amostras foram embutidas em resina epóxi, polidas e posteriormente recobertas com carbono para torná- 
las condutora de eletricidade (condição necessária para análise ao MEV). Foi utilizado em equipamento de MEV marca LEO, modelo Stereoscan 440, com espectrômetro de raios X por dispersão de energia (EDS) INCA, marca Oxford, com detector de Si. A rotina de trabalho compreendeu a coleta de imagens com detector de elétrons retroespalhados (BSE) e a seleção de pontos ou áreas para microanálise química semi-quantitativa por EDS, para a caracterização composicional.

\section{RESULTADOS E DISCUSSÃO}

$\mathrm{Na}$ metodologia de análise dos resultados foram avaliadas curvas TL de diferentes amostras $\left(Q_{2}\right.$ a $Q_{5}$ ) para a dose de 5 Gy (Figura 1a). Com o objetivo de realizar as análises com o GlowFit com uma maior qualidade, foram utilizadas médias das curvas experimentais, obtendo a curva TL apresentada na Figura 1b.

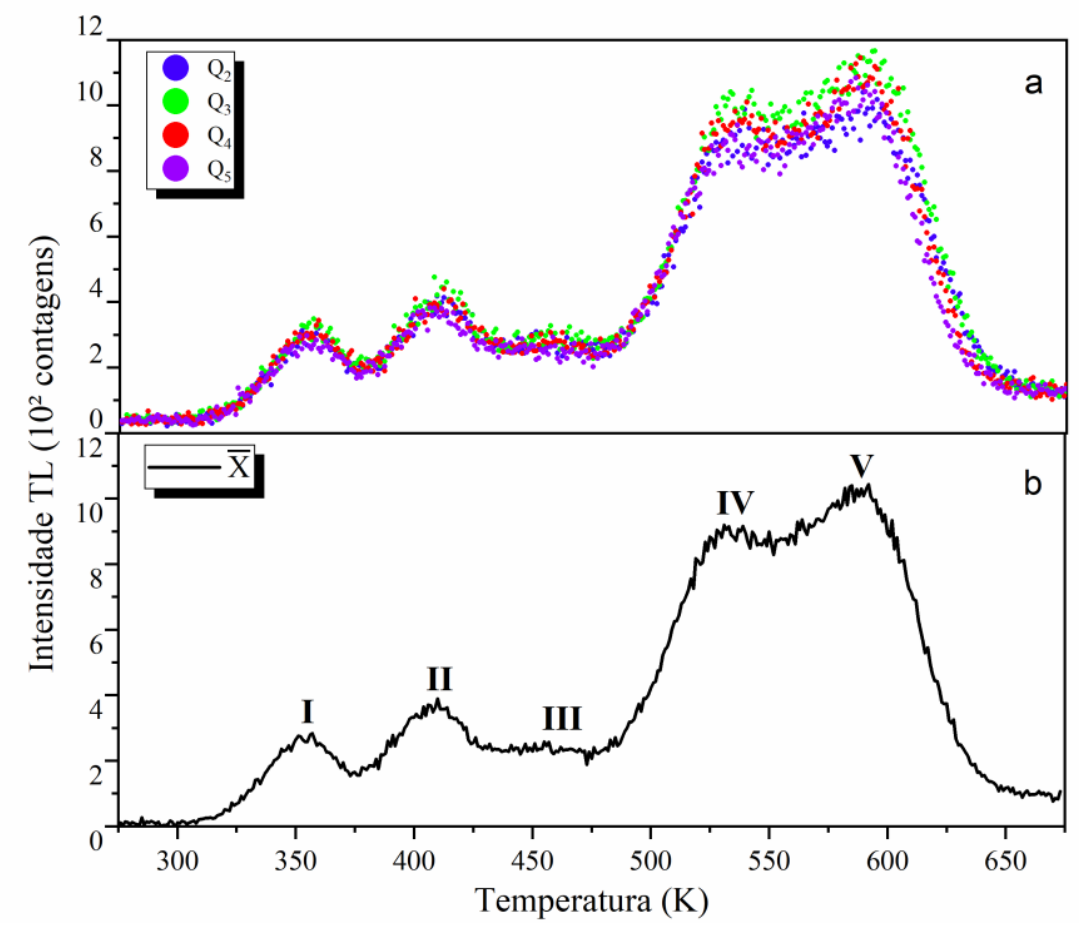

Figura 1: (a) Curvas experimentais para as amostras $Q_{2}$ a $Q_{5}$; (b) Curva média das curvas experimentais, irradiadas com dose beta de $5 \mathrm{~Gy}$.

Na Figura 1, é possível observar a presença de 5 picos termoluminescentes para alexandrita, localizados nas posições de temperatura $355 \mathrm{~K}$ (pico I), $405 \mathrm{~K}$ (pico II), $445 \mathrm{~K}$ (pico III), $535 \mathrm{~K}$ (pico IV) e $585 \mathrm{~K}$ (pico V), valores próximos aos observados da literatura [24,25,36]. Ainda na Fi- 
gura 1a, apesar de apresentar alguns ruídos nas curvas experimentais, é possível observar que os sinais TL das quatro amostras analisadas têm valores muito próximos, resultando numa média dos pontos experimentais apresentado na Figura 1b. Para fins de aplicações dosimétricas, nesse trabalho a análise é feita nos picos mais intensos e estáveis, denominados IV e V. Os parâmetros analisados nas curvas TL foram linearidade, repetibilidade e fading.

Primeiramente, com o objetivo de caracterizar detalhadamente a natureza da curva de emissão, assim como verificar a ordem cinética, o método $T_{M}-T_{S T O P}$ (Figura 2a) foi realizado com foco nos picos denominados IV e V. O método $T_{M}-T_{S T O P}$ foi realizado na amostra $Q_{6}$ utilizando dose beta de 5 Gy e intervalo de $T_{\text {STOP }}$ de $10 \mathrm{~K}$, sendo pré-aquecida de 443 a $673 \mathrm{~K}$. Foram obtidas 15 curvas residuais, resultando no gráfico da Figura $2 \mathrm{a}$. O gráfico $T_{M}-T_{S T O P}$ também demonstra que existem 2 picos distintos que compõe o trecho da curva estudado, como pode ser visto na Figura $2 \mathrm{~b}$.

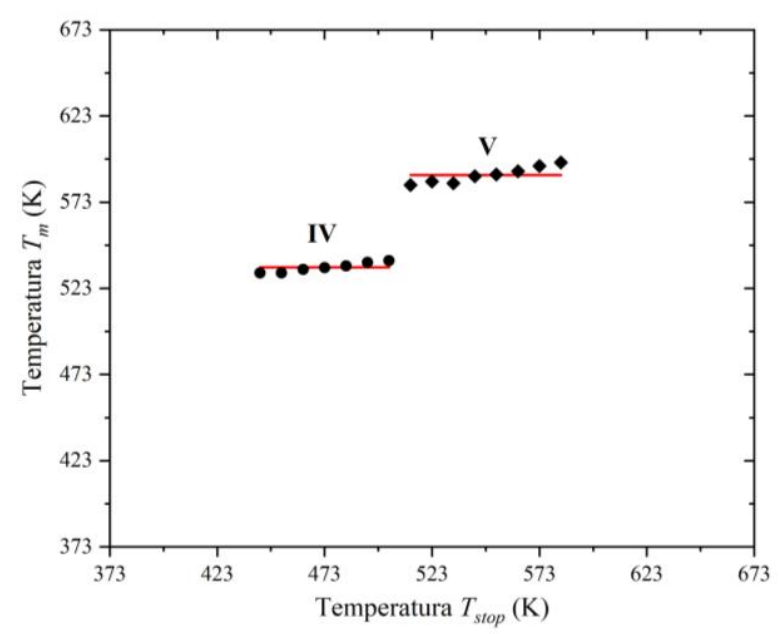

a

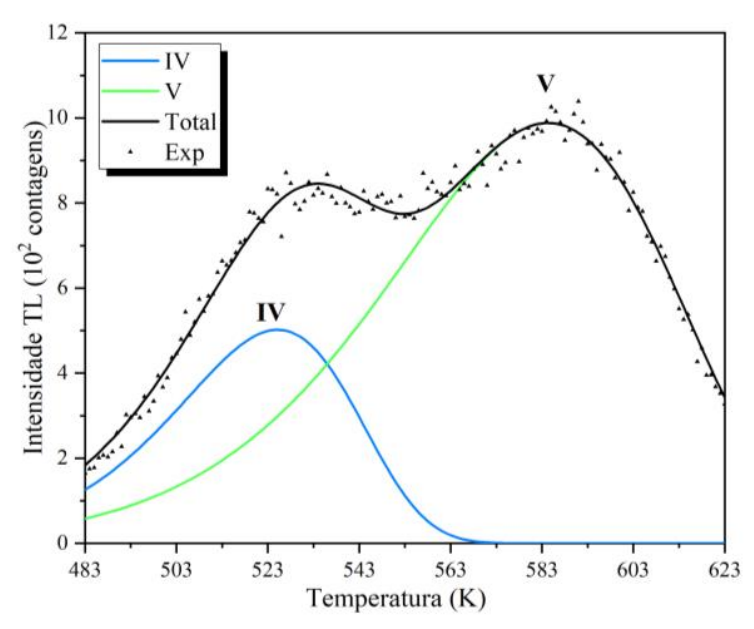

b

Figura 2: (a) Resultado do $T_{M}-T_{S T O P}$ de 15 curvas residuais para dose beta de 5 Gy. (b) Picos dosimétricos ajustados a partir dos intervalos obtidos no $T_{M}-T_{S T O P}\left(\right.$ curva residual de $T_{S T O P}=483 \mathrm{~K}$ ), com parâmetro $F O M=4,01 \%$.

Os resultados $T_{M}-T_{S T O P}$ mostraram que a média da posição de temperatura do pico IV foi de $535,1 \pm 2,7 \mathrm{~K}$, e para o pico V foi de 588,7 $\pm 4,7 \mathrm{~K}$. O perfil de "degraus" observado na Figura 2 a é coerente com o modelo que descreve diversos picos TL com o mecanismo cinético de primeira ordem [14]. Portanto, a partir desses dados, as análises da curva TL, utilizando o software Glowfit, foram baseadas na deconvolução da curva com dois picos, conforme mostrado na Figura $2 b$. 
O experimento de dose-resposta consistiu em observar o comportamento das curvas TL obtidas da alexandrita em relação às variações de doses recebidas pelas amostras. As curvas TL foram obtidas para doses entre 0,5 e 5 Gy. O resultado pode ser observado na Figura 3a. Com o ajuste de curvas do GlowFit, foi possível obter as temperaturas $\left(T_{M}\right)$ referentes a cada um dos picos, resultado apresentado na Figura 3b.

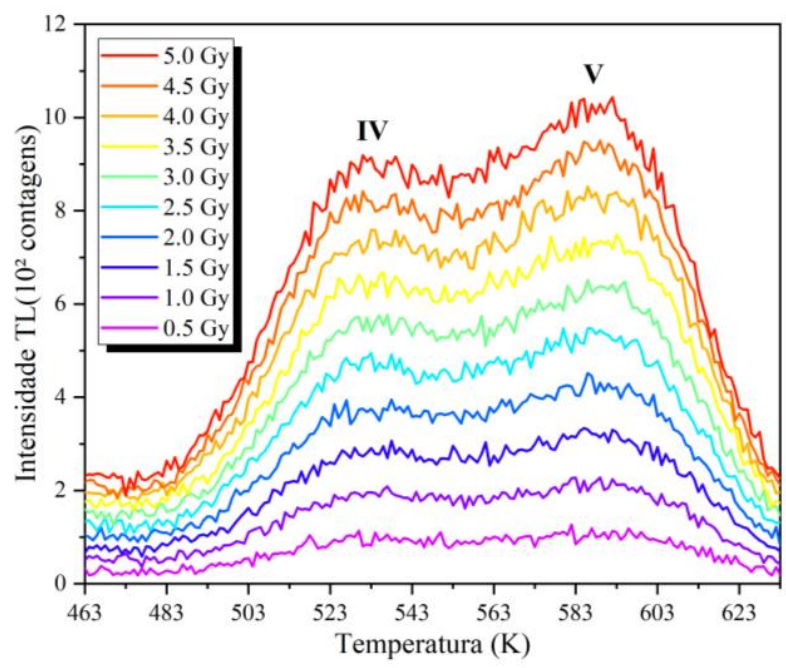

a

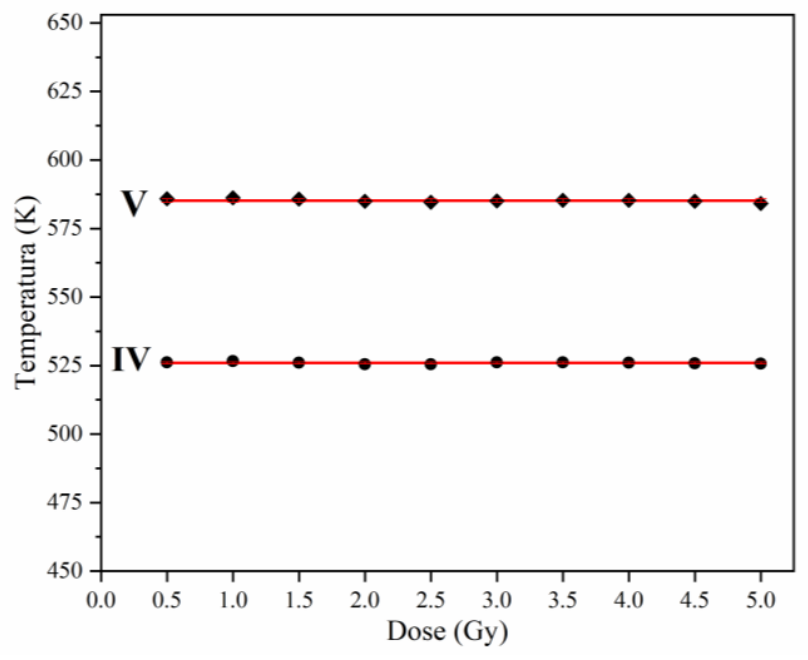

$\mathrm{b}$

Figura 3: (a) Variação da intensidade das curvas TL experimentais para doses beta (0,5-5 Gy). (b) Posição dos picos em função da dose.

Observou-se que o parâmetro figure of merit (FOM) para as curvas TL atenderam a um bom ajuste, estando próximo ou abaixo de 5\% [32-35]. Também foram obtidos, pelo software GlowFit, as energias de ativação $E$, e o fator frequência $s$ para cada um dos picos ajustados em função da dose. O valor médio e o desvio padrão dos dados obtidos são apresentados na Tabela 2.

Tabela 2: Média dos valores de energia $(E)$ e fator de frequência $(s)$ obtido a partir das curvas TL em função da dose (0,5 - 5 Gy).

\begin{tabular}{ccc}
\hline Pico & $\boldsymbol{E}(\mathbf{e V})$ & $\boldsymbol{s}\left(\mathbf{s}^{-\mathbf{1}}\right)$ \\
\hline IV & $1,130 \pm 0,018$ & $(3,2 \pm 1,1) \times 10^{13}$ \\
V & $0,828 \pm 0,014$ & $(3,8 \pm 1,4) \times 10^{9}$ \\
\hline
\end{tabular}

No experimento dose-resposta, é possível observar que conforme há o aumento da dose, a curva TL apresenta um aumento da área total, como pode ser visto na Figura 3a. Também é possível ob- 
servar, na Figura 3b, que os valores de $T_{M}$ são aproximadamente constantes em função da dose, portanto, reforçando que os picos TL (IV e V) da alexandrita obedecem a um mecanismo cinético de primeira ordem. Na Tabela 3, é possível observar que o fator frequência $s$ para o pico V está abaixo do fator de vibração da rede cristalina estimado, que seria entre $10^{12}$ e $10^{14}$ [37]. De acordo com Chen e colaboradores [38], uma das possíveis razões para que essa diferença ocorra se dá quando as curvas ajustadas através de um método numérico, como o GlowFit, são realizadas para picos muito próximos uns dos outros, gerando um aparente valor anômalo para $s$. De fato, os picos IV e V são picos muito próximos, havendo em parte uma sobreposição entre eles.

Também foram analisadas as integrais de cada pico em função da dose de radiação, apresentadas na Figura 4. Os dados da linearização estão na Tabela 3.

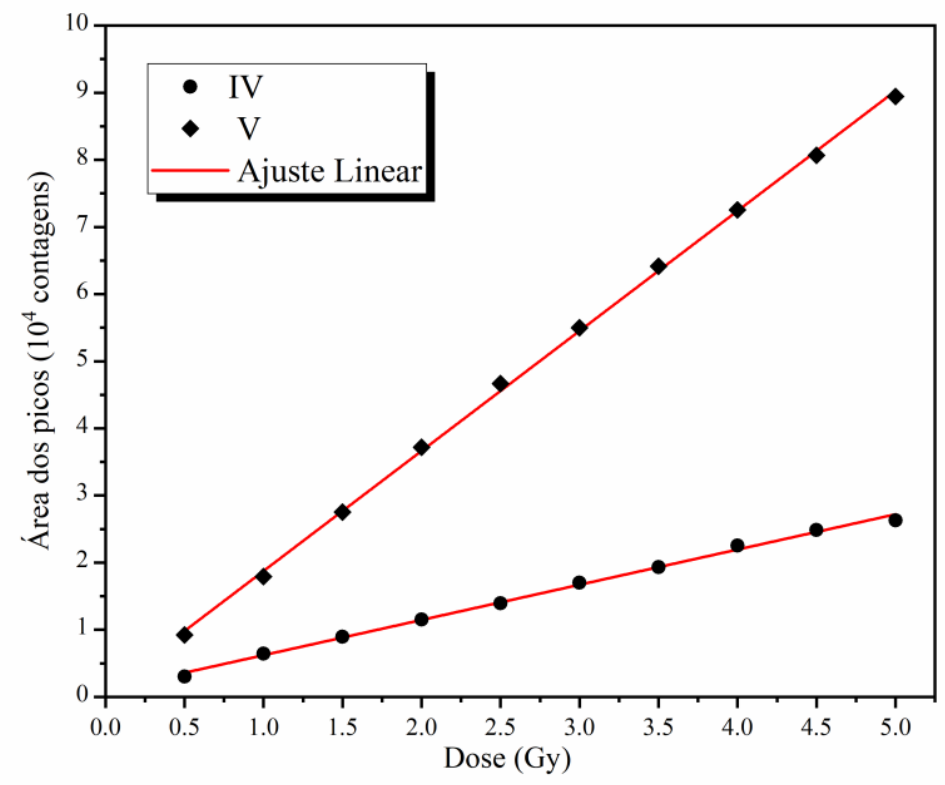

Figura 4: Integrais dos picos TL para as curvas médias ajustadas das amostras $Q_{2}$ a $Q_{5}$.

Tabela 3: Ajuste linear da dose-resposta quanto aos parâmetros $b$ (coeficiente linear), $a$ (coeficiente angular) e $R^{2}$ (coeficiente de determinação).

\begin{tabular}{|c|c|c|c|}
\hline Picos & $b \cdot\left(10^{2}\right.$ contagens $)$ & $a \cdot\left(10^{3}\right.$ contagens $\left.\mathrm{Gy}^{-1}\right)$ & $R^{2}$ \\
\hline IV & $9,6 \pm 3,1$ & $5,25 \pm 0,10$ & 0,99708 \\
\hline $\mathrm{V}$ & $8,6 \pm 5,0$ & $17,88 \pm 0,16$ & 0,99934 \\
\hline
\end{tabular}

A Figura 4 mostrou que o sinal TL possui relação linear com a dose de radiação beta, observado 
pelos coeficientes de determinação $R^{2}$ próximos de 1, para as áreas tanto dos picos IV, como o $\mathrm{V}$. Esse fator mostra que a linearidade da dose-resposta na faixa de 0,5 Gy a 5 Gy é satisfatória, o que caracteriza uma das qualidades desejadas para a aplicação do material na área da dosimetria.

A partir das áreas de cada pico TL, obtido pelo software GlowFit, foi feita uma análise estatística da repetibilidade observando a média, o desvio padrão e o coeficiente de variação entre as medidas, resultando na construção do gráfico na Figura 5, assim como a Tabela 4 contendo análises desses resultados.

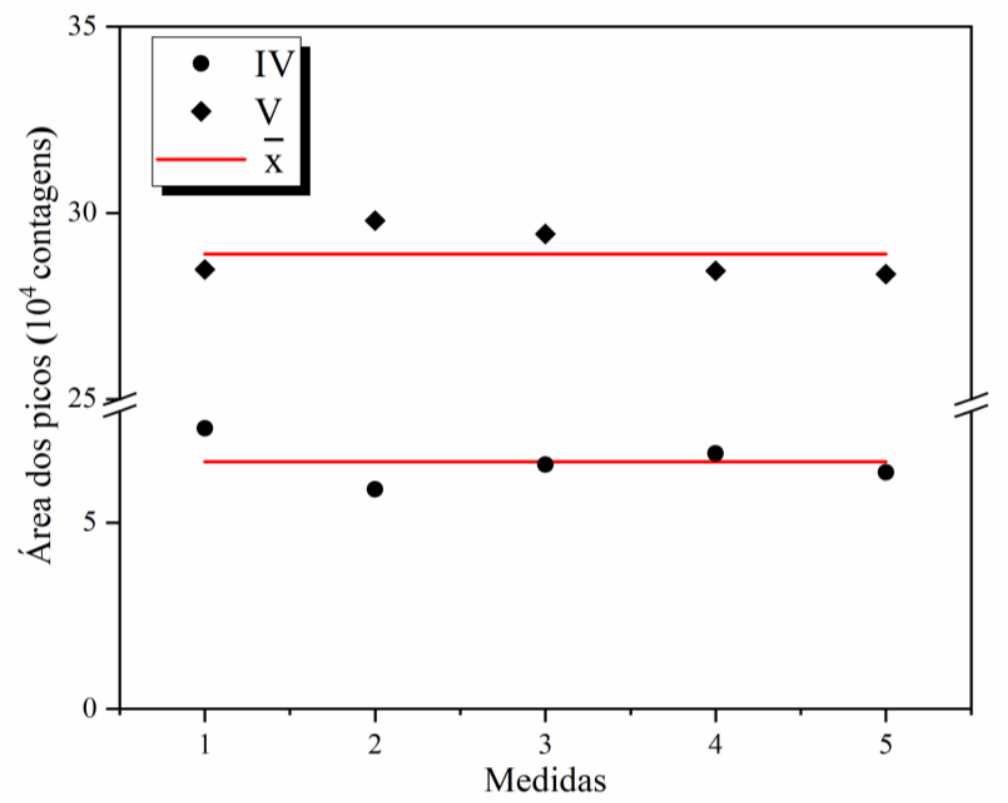

Figura 5: Gráfico da repetibilidade do sinal TL, sendo que os pontos são as integrais dos picos TL (IV e V); e a linha contínua é a média aritmética simples dos dados obtidos para cada um dos picos $T L$.

Tabela 4: Dados da repetibilidade obtidos quanto a média das integrais para as 5 medidas $(\bar{x})$, o desvio padrão $(\sigma)$ e o coeficiente de variação $(C V)$.

\begin{tabular}{cccc}
\hline Dados & $\bar{x}$ & $\boldsymbol{\sigma}$ & $\boldsymbol{C} \boldsymbol{V}(\boldsymbol{\%})$ \\
\hline IV & $6,64 \times 10^{3}$ & $5,45 \times 10^{2}$ & 8,20 \\
V & $2,89 \times 10^{4}$ & $5,92 \times 10^{2}$ & 2,05 \\
\hline
\end{tabular}

No experimento de repetibilidade, é possível observar que os picos TL tiveram um valor aproximadamente constante independentemente do número de medidas. É importante mencionar que o 
valor da intensidade para esses resultados (dose beta de 1 Gy) é maior em relação às medidas observadas na Figura 4, pois a amostra utilizada no experimento de repetibilidade tem uma massa consideravelmente maior (Tabela 1) do que as utilizadas na análise dose-resposta. Os resultados na Tabela 4 demonstraram um valor baixo para o coeficiente de variação para os picos, portanto, conclui-se que os valores das áreas das curvas TL demonstram ser reprodutíveis, para medidas realizadas sob as mesmas condições.

O experimento do fading foi realizado utilizando a amostra $Q_{1}$, com um tempo de armazenamento em temperatura ambiente de 0 min., 2 dias e 33 dias, mostrado na Figura 6. Para as irradiações, foi utilizada uma dose beta de 5 Gy. As curvas foram ajustadas no GlowFit, então os valores das áreas dos picos IV e V foram obtidos e normalizados em relação a curva obtida imediatamente após a exposição à radiação ionizante (Tabela 6).

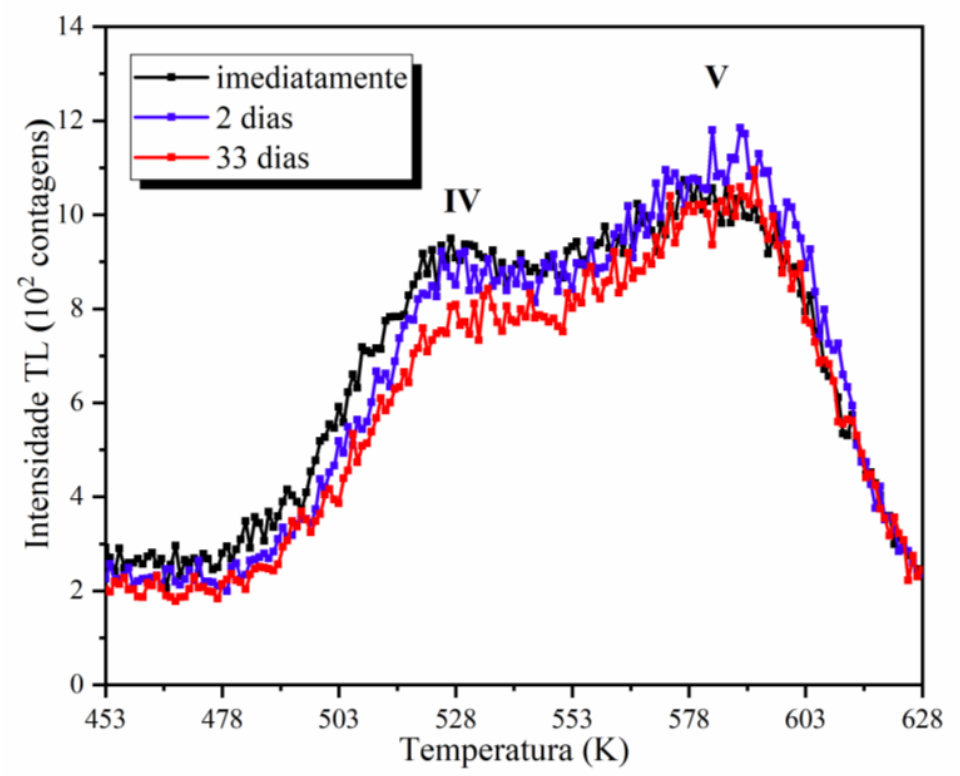

Figura 6: Gráfico de fading do sinal TL realizado após o período de até 33 dias com radiação ionizante prévia de 5 Gy.

Tabela 5: Normalização da integral dos picos dosimétricos para diferentes períodos de armazenamento (fading), com a perda em \% do sinal TL em relação a medida imediata após a exposição à radiação beta (5 Gy).

\begin{tabular}{ccc}
\hline Dados & 2 dias (\%) & 33 dias (\%) \\
\hline IV & 0,00 & 7,46 \\
V & 0,00 & 6,12 \\
\hline
\end{tabular}


Quanto ao fading do sinal TL (Figura 6) nota-se que os picos IV e V obtiveram um decaimento mais relevante apenas para o período de 33 dias, conforme mostra a Tabela 5. Ainda assim, o fading ficou abaixo de $10 \%$ do sinal TL inicial e muito próximos das variações obtidas por repetibilidade (Tabela 4). Portanto, os resultados de repetibilidade e fading, indicam que, no geral, os picos IV e V mantém e reproduzem o sinal luminescente, propriedade desejável para um material dosimétrico [39].

Complementarmente, a alexandrita foi caracterizada por MEV-EDS. Na amostra preparada com a resina epóxi, as medidas foram tomadas para duas regiões distintas de sua superfície, mostradas na Figura 7. A partir da imagem resultante das regiões A e B, foram analisados dois espectros de microanálise química (EDS), escolhendo pontos em diferentes grãos ou fases para a análise. A composição química semiquantitativa determinada por MEV-EDS estão dispostos nas Tabela 6 (região A) e Tabela 7 (região B).

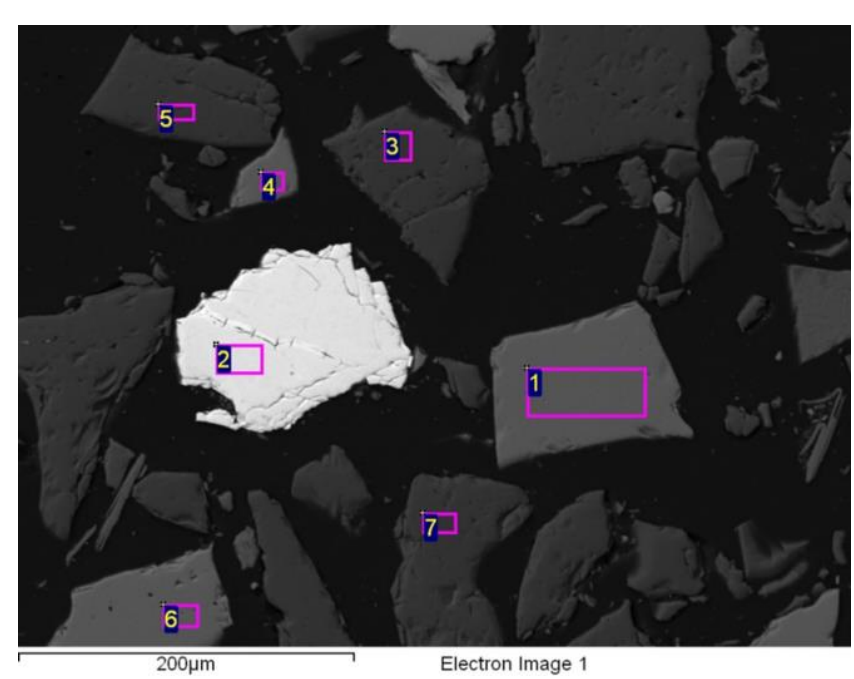

a

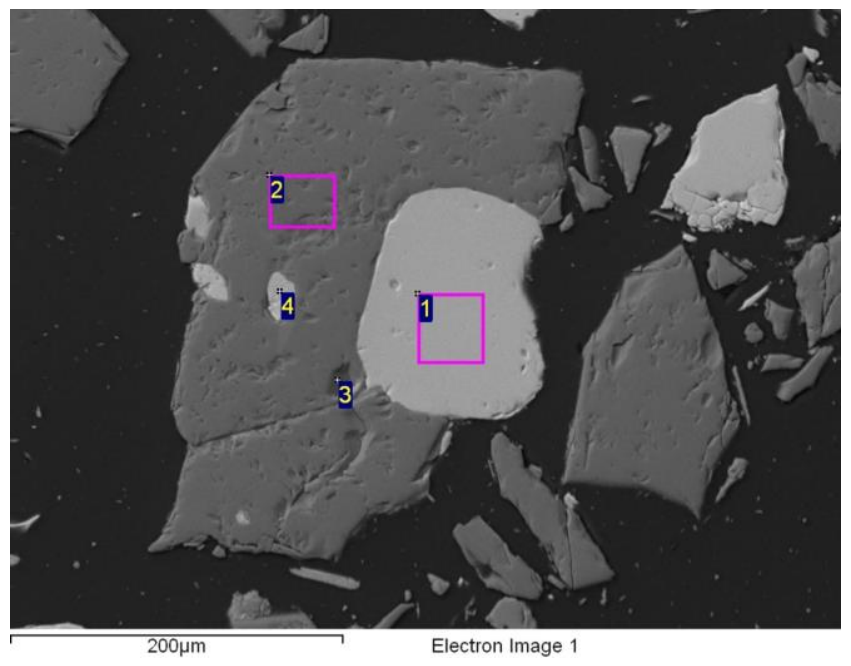

b

Figura 7: Microscopia eletrônica de varredura da região (a) A e (b) B. 
Tabela 6: Resultados do EDS para a região A, em wt\%.

\begin{tabular}{cccccccccccccccc}
\hline Pontos & $\mathbf{O}$ & $\mathbf{F}$ & $\mathbf{N a}$ & $\mathbf{A l}$ & $\mathbf{S i}$ & $\mathbf{P}$ & $\mathbf{C a}$ & $\mathbf{T i}$ & $\mathbf{C r}$ & $\mathbf{F e}$ & $\mathbf{N b}$ & $\mathbf{T a}$ & $\mathbf{U}$ & Total & Be$^{*}$ \\
\hline 1 & 41,4 & 3,7 & -- & -- & -- & 18,2 & 36,6 & -- & -- & -- & -- & -- & -- & 100,0 & -- \\
2 & -- & -- & 0,7 & -- & 2,4 & -- & 11,8 & 2,2 & -- & 1,4 & 13,8 & 27,8 & 6,1 & 66,4 & -- \\
3 & 50,0 & -- & -- & 41,2 & -- & -- & -- & -- & 0,93 & 1,02 & -- & -- & -- & 93,2 & 6,8 \\
4 & 40,7 & 4,4 & -- & -- & -- & 18,3 & 36,6 & -- & -- & -- & -- & -- & -- & 100,0 & -- \\
5 & 49,6 & -- & -- & 41,9 & -- & -- & -- & -- & 0,8 & 1,3 & -- & -- & -- & 93,5 & 6,5 \\
6 & 40,2 & 4,3 & -- & -- & -- & 18,4 & 37,1 & -- & -- & -- & -- & -- & -- & 100,0 & -- \\
7 & 49,2 & -- & -- & 42,4 & -- & -- & -- & -- & 1,5 & 1,1 & -- & -- & -- & 94,1 & 5,9 \\
\hline
\end{tabular}

*Berílio foi estimado a partir da diferença do valor total (100\%).

Tabela 7: Resultados do EDS para a região B, em wt\%.

\begin{tabular}{cccccccccc}
\hline Pontos & O & F & Al & P & Ca & Cr & Fe & Total & Be* $^{*}$ \\
\hline 1 & 41,0 & 4,2 & -- & 18,2 & 36,6 & -- & -- & 100,0 & -- \\
2 & 49,4 & -- & 42,5 & -- & -- & 0,9 & 1,2 & 93,9 & 6,1 \\
3 & 46,5 & -- & 51,2 & -- & -- & 0,8 & 1,4 & 99,9 & 0,1 \\
4 & 39,7 & 4,5 & -- & 18,5 & 37,4 & -- & -- & 100,0 & -- \\
\hline
\end{tabular}

*Berílio foi estimado a partir da diferença do valor total (100\%).

De maneira geral, nas observações no MEV-EDS, o pó de alexandrita utilizado apresentou distribuição homogênea dos grãos. Na região A (Figura 7a) é possível observar uma predominância de grãos caracterizados como alexandrita nos pontos 3, 5 e 7. Também existem grãos de apatita nos pontos 1, 4 e 6, enquanto o ponto 2 não foi identificado. A região B (Figura 7b) possui formação semelhante à região A, com predominância de fases de alexandrita (pontos 2 e 3) e algumas fases contendo apatita (pontos 1 e 4). Dessa forma, é possível observar que, de fato, as amostras trituradas são constituídas predominantemente por alexandrita. As fases de alexandrita também possuem composições semelhantes quanto a porcentagem dos elementos, com exceção do ponto 3 na região B. Nesse caso, por se tratar de uma região muito pequena em relação ao diâmetro do feixe de elétrons, a composição química apresentada reflete também a composição do entorno. Apesar de se tratar de uma análise semiquantitativa, foi identificada a presença do $\mathrm{Cr}$ e do Fe em todas as fases de alexandrita, sendo esses, elementos importantes e responsáveis por muitas das propriedades ópticas $[2,4,36,40]$ e elétricas [41] observadas nesse material. 


\section{CONCLUSÃO}

Neste trabalho foram investigadas as características termoluminescentes do mineral $\mathrm{BeAl}_{2} \mathrm{O}_{4}: \mathrm{Cr}^{3+}$, sendo sua curva TL caracterizada por 5 picos, com suas temperaturas localizadas em $355 \mathrm{~K}$ (pico I), $405 \mathrm{~K}$ (pico II), $445 \mathrm{~K}$ (pico III), $530 \mathrm{~K}$ (pico IV) e $585 \mathrm{~K}$ (pico V). Este trabalho teve como foco os picos IV e V por serem mais intensos e estáveis (altas temperaturas). Com a verificação pelo método $T_{M}-T_{S T O P}$, e pela constância das temperaturas de pico com a dose absorvida, foi possível conferir que os picos IV e V da alexandrita seguem o mecanismo cinético de primeira ordem. Isso permitiu a utilização do software GlowFit, para a deconvolução da curva TL experimental, sendo então feita uma análise mais detalhada dos parâmetros dos picos TL. Foram obtidos a energia de ativação $E_{I V}=1,130 \pm 0,018 \mathrm{eV}$ e $E_{V}=0,828 \pm 0,014 \mathrm{eV}$, e o fator frequência $(s)$ na ordem de $10^{13} \mathrm{~s}^{-1}$ para o pico IV e $10^{9} \mathrm{~s}^{-1}$ para o pico V. Para a variação de doses de 0,5 Gy a $5 \mathrm{~Gy}$, a alexandrita apresentou uma boa linearidade no sinal TL de ambos os picos em função da dose, sendo o coeficiente de determinação $R^{2}$ próximo de 1 . Também foi verificada a boa repetibilidade para medidas realizadas sob as mesmas condições, tendo sido obtido um $C V<10 \%$ para ambos os picos TL (IV e V). Os mesmos picos também tiveram um desvanecimento (fading) $<10 \%$ no período de armazenamento de 33 dias, demonstrando que os picos IV e V são estáveis à temperatura ambiente. É provável que os picos de emissão TL estejam relacionados à presença das impurezas como $\mathrm{Cr}^{3+} \mathrm{e}$ $\mathrm{Fe}^{3+}$, observados na caracterização química no mineral, e que são os principais responsáveis pelas propriedades ópticas e elétricas desse material. Em geral, os resultados TL mostraram algumas das características desejáveis dos materiais dosimétricos, sugerindo que a alexandrita tem potencial para aplicação nessa área.

\section{AGRADECIMENTOS}

S. L. Dardengo (\#2018/22179-6), M. C. S. Nunes (\#2018/16894-4) e N. M. Trindade (\#2019/05915-3) agradecem a Fundação de Amparo à Pesquisa do Estado de São Paulo (FAPESP). E. M. Yoshimura é agradecida ao Conselho Nacional de Desenvolvimento Científico e Tecnológico (CNPq), \#306843/2018-8, e à FAPESP, \#2018/05982-0. Este trabalho foi parcialmente apoiado 
pelo Laboratório de Caracterização Tecnológica da USP (LCT-USP), no qual agradecemos os geólogos Marco Timich e Renato Contessotto.

\section{REFERÊNCIAS}

[1] PETERSEN JÚNIOR, K. J.; SCHULTZ-GÜTTLER, R. A. Alexandrita no município de Minaçu, Goiás: Mineralogia, Geologia e considerações genéticas. Dissertação (Mestrado em Mineralogia e Petrologia) - Instituto de Geociências, Universidade de São Paulo. São Paulo, 186 p. 1998.

[2] SCALVI, R. M. F. Relaxação dilpolar elétrica fotoinduzida em alexandrita sintética e natural. Tese (Doutorado em Ciência e Engenharia de Materiais). Ciência e Engenharia de materiais - Universidade de São Paulo. São Carlos, SP. 154 p. 2000.

[3] BUKIN, G. V. Optical generation in alexandrite $\left(\mathrm{BeAl}_{2} \mathrm{O}_{4}: \mathrm{Cr}^{3+}\right)$. Kvant Tovaya Electronika, v. 5, p. 1168-1169, 1978.

[4] TRINDADE, N. M.; SCALVI, R. M. F. Investigação das propriedades ópticas e elétricas em alexandrita natural e sintética. Dissertação (Mestrado em Ciência e Tecnologia de Materiais). Universidade Estadual Paulista "Julio de Mesquita Filho". Bauru, SP. 175 p. 2009.

[5] TOOSI, S. et al. Treatment of trichostasis spinulosa with a 755-nm long-pulsed alexandrite laser. Journal of the European Academy of Dermatology and Venereology, v. 24, n. 4, p. 470473, 2010.

[6] IBRAHIMI, O. A. et al. Laser hair removal. Dermatologic Therapy, v. 24, n. 1, p. 94-107, 2011.

[7] NILFOROUSHZADEH, M. A. et al. Comparison Between Sequentional Treatment With Diode and Alexandrite Lasers Versus Alexandrite Laser Alone in the Treatment of Hirsutism. J Drugs Dermatol., v. 10, n. 11, p. 1255-1259, 2011.

[8] LI, Y. et al. Q-switched alexandrite laser treatment of facial and labial lentigines associated with Peutz-Jeghers syndrome. Photodermatology, Photoimmunology \& Photomedicine, v. 28, n. 4, p. 196-199, 2012.

[9] WANG, Y.; QIAN, H.; LU, Z. Treatment of café au lait macules in Chinese patients with a Qswitched 755-nm alexandrite laser. Journal of Dermatological Treatment, v. 23, n. 6, p. 431-436, 
2012.

[10] KIM, Y. K. et al. Therapeutic efficacy of long-pulsed 755-nm alexandrite laser for seborrheic keratoses. Journal of the European Academy of Dermatology and Venereology, v. 28, n. 8, p. 1007-1011, 2014.

[11] MALÍČKOVÁ, I. et al. Laser effect in the optical luminescence of oxides containing Cr. Acta Geologica Slovaca, v. 10, n. 1, p. 27-34, 2018.

[12] LALIC, S. S. et al. Novos materiais dosimétricos para aplicações em física médica. Revista Brasileira de Física Médica, v. 13, n. 1, p. 10, 2019.

[13] YUKIHARA, E. G.; MCKEEVER, S. W. S. Optically Stimulated Luminescence: Fundamentals and Applications. West Sussex: UK: John Wiley and Sons, 2011.

[14] MCKEEVER, S. W. S. Thermoluminescence of Solids. Cambridge: Cambridge University Press, 1985.

[15] KALITA, J. M.; WARY, G. Thermoluminescence properties of minerals and their application. Saarbrucken, Germany: LAP LAMBERT Academic Publishing, 2016.

[16] SOUZA, S.; YAMAMOTO, T.; D'ERRICO, F. Estado da arte em dosimetria do estado solido. International Joint Conference RADIO 2014. Anais... Gramado: 2014

[17] CORRECHER, V. et al. Luminescence characterization of a sodium-rich feldspar. Radiation Effects \& Defects in Solids, v. 162, n. 10-11, p. 709-714, 2007.

[18] KIBAR, R. et al. Luminescent, optical and color properties of natural rose quartz. Radiation Measurements, v. 42, n. 10, p. 1610-1617, 2007.

[19] OGUNDARE, F. O.; CHITHAMBO, M. L. Thermoluminescence kinetic analysis of quartz with a glow peak that shifts in an unusual manner with irradiation dose. Journal of Physics D: Applied Physics, v. 40, n. 1, p. 247-253, 2007.

[20] PREUSSER, F. et al. Quartz as a natural luminescence dosimeter. Earth-Science Reviews, v. 97, n. 1, p. 184-214, 2009.

[21] ABDEL-RAZEK, Y. A. Thermoluminescence dosimetry using natural calcite. Journal of Taibah University for Science, v. 10, n. 2, p. 286-295, 2016.

[22] LIMA, J. F. DE; VALERIO, M. E. G.; OKUNO, E. Thermally assisted tunneling: An alternative model for the thermoluminescence process in calcite. Physical Review B, v. 64, n. 1, p. 14105, 2001. 
[23] PANDYA, A.; VAIJAPURKAR, S. G.; BHATNAGAR, P. K. Radiation dosimetry by potassium feldspar. Bulletin of Materials Science, v. 23, n. 2, p. 155-158, 2000.

[24] TRINDADE, N. M. et al. Thermoluminescence and radioluminescence of alexandrite mineral. Journal of Luminescence, v. 206, 2019.

[25] TRINDADE, N. M.; KAHN, H.; YOSHIMURA, E. M. Thermoluminescence of natural $\mathrm{BeAl}^{2} \mathrm{O}^{4}: \mathrm{Cr}^{3+}$ Brazilian mineral: Preliminary studies. Journal of Luminescence, v. 195, 2018.

[26] IVANOV, V. Y. et al. Electronic excitations in $\mathrm{BeAl}_{2} \mathrm{O}_{4}, \mathrm{Be}_{2} \mathrm{SiO}_{4}$, and $\mathrm{Be}_{3} \mathrm{Al}_{2} \mathrm{Si}_{6} \mathrm{O}_{18}$ crystals. Physics of the Solid State, v. 47, n. 3, p. 466-473, 2005.

[27] GROPPO, D. P.; CALDAS, L. V. E. Luminescent response from BeO exposed to alpha, beta and X radiations. Radiation Measurements, v. 71, n. Supplement C, p. 81-85, 2014.

[28] AZORIN NIETO, J. Present status and future trends in the development of thermoluminescent materials. Applied Radiation and Isotopes, v. 117, p. 135-142, 2016.

[29] MCKEEVER, S. W. S. On the analysis of complex thermoluminescence. Glow-curves: Resolution into individual peaks. physica status solidi (a), v. 62, n. 1, p. 331-340, 1980.

[30] PUCHALSKA, M.; BILSKI, P. GlowFit - a new tool for thermoluminescence glow-curve deconvolution. Radiation Measurements, v. 41, n. 6, p. 659-664, 2006.

[31] RANDALL, J. T.; WILKINS, M. H. F. Phosphorescence and electron traps - I. The study of trap distributions. Proceedings of the Royal Society of London. Series A. Mathematical and Physical Sciences, v. 184, n. 999, p. 365, 1945.

[32] SHARMA, B. A.; SINGH, T. B.; GARTIA, R. K. Critical evaluation of goodness of fit of computerised glow curve deconvolution. Indian Journal of Pure \& Applied Physics, v. 42, p. 492-497, 2004.

[33] PRADHAN, A. S. et al. TL glow curve shape and response of LiF:Mg,Cu,Si-Effect of heating rate. Radiation Measurements, v. 43, n. 2-6, p. 361-364, Feb. 2008.

[34] DELICE, S.; BULUR, E.; GASANLY, N. M. Anomalous heating rate dependence of thermoluminescence in T12GaInS4 single crystals. Journal of Materials Science, v. 49, n. 24, p. 8294-8300, Dec. 2014.

[35] GINTHER, R. J.; KIRK, R. D. The Thermoluminescence of $\mathrm{CaF}_{2}: \mathrm{Mn}$. Journal of The Electrochemical Society, v. 104, n. 6, p. 365, Jun. 1957.

[36] FERRAZ, G. M. et al. TL, EPR and Optical Absorption Studies on Natural Alexandrite 
Compared to Natural Chrysoberyl. Radiation Protection Dosimetry, v. 100, n. 1-4, p. 471-474, 2002.

[37] YUKIHARA, E. G.; OKUNO, E. Desvendando a cor e a termoluminescência do topázio: um estudo dos defeitos e processos termicamente e opticamente estimulados no cristal natural. Tese (Doutorado em Ciências). Instituto de Física, Departamento de Física Nuclear - Universidade de São Paulo. São Paulo. 380 p. 2001.

[38] CHEN, R.; PAGONIS, V.; LAWLESS, J. L. Evaluated thermoluminescence trapping parameters-What do they really mean? Radiation Measurements, v. 91, p. 21-27, 2016.

[39] PEKPAK, E.; YILMAZ, A.; OZBAYOGLU, G. An Overview on Preparation and TL Characterization of Lithium Borates for Dosimetric Use. The Open Mineral Processing Journal, v. 3, n. 1, p. 14-24, May 2010.

[40] PUGH-THOMAS, D.; WALSH, B. M.; GUPTA, M. C. Spectroscopy of $\mathrm{BeAl}_{2} \mathrm{O}_{4}: \mathrm{Cr}^{3+}$ with application to high-temperature sensing. Applied Optics, v. 49, n. 15, p. 2891-2897, 2010.

[41] TRINDADE, N. M. et al. Photo-Induced Thermally Stimulated Depolarization Current (TSDC) in Natural and Synthetic Alexandrite $\left(\mathrm{BeAl}_{2} \mathrm{O}_{3}: \mathrm{Cr}^{3+}\right)$. Materials Sciences and Applications, v. 7, n. 12, p. 881-894, 2016. 